\title{
Comparison of Filter Line Search Algorithms in the Primal-Dual Barrier Approach for Nonlinear Programming
}

\author{
M. Fernanda P. Costa* and Edite M.G.P. Fernandes ${ }^{\dagger}$ \\ ${ }^{*}$ Department of Mathematics for Science and Technology, University of Minho, Portugal \\ ${ }^{\dagger}$ Department of Production and Systems, University of Minho, 4710-057 Braga, Portugal
}

\begin{abstract}
In this paper, we present a new filter line search method based on two measures that is integrated into the primaldual barrier method developed by Wächter and Biegler [Mathematical Programming 106 (2006), pp. 25-57] for nonlinear programming. One measure arises directly from the first order optimality conditions of the problem and the other is the barrier function. Primary assessment of the method has been done with a well-known collection of problems and compared with the solver IPOPT.
\end{abstract}

Keywords: nonlinear programming, barrier method, filter method

PACS: $02.60 . P n$

\section{INTRODUCTION}

The optimization problem to be addressed in this paper is written in the form:

$$
\begin{array}{rl}
\min _{x \in \mathbb{R}^{n}} & f(x) \\
\text { subject to } & c(x)=0 \\
& x \geq 0,
\end{array}
$$

where $c_{i}: \mathbb{R}^{n} \rightarrow \mathbb{R}$ for $i=1, \ldots, m$ and $f: \mathbb{R}^{n} \rightarrow \mathbb{R}$ are nonlinear and twice continuously differentiable functions. The inequality constraints can be rewritten as equality constraints by introducing slack variables.

Interior point methods based on a logarithmic barrier function have been widely used for nonlinear programming $[8,10]$. To allow convergence from poor starting points, barrier and augmented Lagrangian merit functions may be used [7]. Some line search frameworks use penalty merit functions to enforce progress toward the solution. As an alternative to merit functions, Fletcher and Leyffer [5] proposed a filter method as a tool to guarantee global convergence in algorithms for nonlinear optimization. This technique incorporates the concept of nondominance to build a filter that is able to accept trial points if they improve either the objective function or the constraints violation, instead of a combination of those two measures defined by a merit function. The filter replaces the use of merit functions, so avoiding the update of penalty parameters that are associated with the penalization of the constraints in a merit function. The filter technique has already been adapted to interior point methods. In [11, 12, 13], a filter line search strategy incorporated in a barrier type method is used. The two components of each entry in the filter are the barrier objective function and the constraints violation. In [9], a two-dimensional filter is used in a primal-dual interior point method context. The two entries, measuring quasi-centrality and optimality, combine the three criteria of the first order optimality conditions. A three-dimensional filter based line search strategy has already been tested in [1,2]. The three components of the filter measure feasibility, centrality and optimality and are present in the first order KKT conditions of the barrier problem associated with the problem (1). The optimality measure relies on the norm of the gradient of the Lagrangian function. Convergence to stationary points may be proved, although convergence to a local minimizer is not guaranteed. In [3], the feasibility and centrality measures are maintained, but the barrier objective function is used as the optimality measure.

In this paper we propose a new filter line search method to incorporate into the primal-dual barrier approach of Wächter and Biegler $[11,12,13]$. The three criteria of the first order optimality conditions are combined to define the first measure, herein denoted as KKT error, and the objective function of the associated barrier problem is the other measure, so that convergence to a stationary point that is a minimizer is guaranteed. Performance assessment of the proposed filter framework, when integrated into the IPOPT code freely available at https://projects.coin-or.org/Ipopt, is carried out with a benchmark set of problems. A comparison with the original IPOPT is presented. 


\section{THE PRIMAL-DUAL BARRIER APPROACH}

This section briefly describes the primal-dual barrier method for solving (1). The solution $x^{*}$ is obtained by computing approximate solutions of a sequence of (associated) barrier problems

$$
\begin{array}{cl}
\min _{x \in \mathbb{R}^{n}} & \Phi(x, \mu) \\
\text { subject to } & c(x)=0
\end{array}
$$

for a decreasing sequence of positive barrier parameters $\mu \searrow 0$, while maintaining $x>0$, where

$$
\Phi(x, \mu)=f(x)-\mu \sum_{i=1}^{n} \log \left(x_{i}\right)
$$

is the barrier function. To solve the problem (2) is equivalent to solving the primal-dual system

$$
\begin{aligned}
\nabla f(x)-\nabla c(x) \delta-\lambda & =0 \\
X \Lambda e-\mu e & =0 \\
c(x) & =0
\end{aligned}
$$

where $\delta$ is the multiplier vector of $c(x)=0, \lambda$ is the multiplier vector associated with the original vector $x \geq 0, \nabla f$ is the gradient vector of $f$ and $\nabla c^{T}$ is the Jacobian matrix of constraints $c, X=\operatorname{diag}\left(x_{i}\right)$ and $\Lambda=\operatorname{diag}\left(\lambda_{i}\right)$ are diagonal matrices and $e \in \mathbb{R}^{n}$ is a vector of all ones. We remark that the system (3), with $\mu=0$, and $x \geq 0$ and $\lambda \geq 0$ are the KKT conditions of problem (1). Applying Newton's method and reducing, the following linear system is obtained to compute the search directions $\Delta x, \Delta \delta$ and $\Delta \lambda$ :

$$
\left[\begin{array}{cc}
-\left(\nabla_{x x}^{2} \mathscr{L}+X^{-1} \Lambda\right) & \nabla c(x) \\
\nabla c(x)^{T} & 0
\end{array}\right]\left[\begin{array}{c}
\Delta x \\
\Delta \delta
\end{array}\right]=\left[\begin{array}{c}
\nabla \Phi(x, \mu)-\nabla c(x) \delta \\
-c(x)
\end{array}\right]
$$

where $\mathscr{L}$ is the Lagrangian function of the problem (2), and

$$
\Delta \lambda=\mu X^{-1} e-\lambda-X^{-1} \Lambda \Delta x
$$

\section{PROPOSED FILTER LINE SEARCH}

For simplicity we use the following notation: $u=(x, \lambda, \delta)$ and $\Delta=(\Delta x, \Delta \lambda, \Delta \delta)$. After the search directions have been computed the proposed filter line search algorithm computes, at each iteration $k$,

$$
x^{(k+1)}=x^{(k)}+\alpha^{(k)} \Delta x^{(k)}, \quad \lambda^{(k+1)}=\lambda^{(k)}+\alpha^{(k)} \Delta \lambda^{(k)}, \quad \delta^{(k+1)}=\delta^{(k)}+\alpha^{(k)} \Delta \delta^{(k)},
$$

where $\alpha^{(k)} \in\left(0, \alpha_{\max }^{(k)}\right]$ is a trial step size that is chosen so that

$$
x^{(k)}+\alpha_{\max }^{(k)} \Delta x^{(k)} \text { and } \lambda^{(k)}+\alpha_{\max }^{(k)} \Delta \lambda^{(k)}
$$

are maintained positive, using a backtracking strategy.

The procedure that decides which trial step size is accepted is a filter method. A filter is a set $\bar{F}_{k}$ that contains pairs of values of $\theta(u)$ and $\Phi(x, \mu)$, that are prohibited for a trial iterate to be acceptable in iteration $k$. These two components of each entry in the filter measure KKT error and optimality, respectively, and they are defined as follows:

$$
\theta(u)=\|\nabla f(x)-\nabla c(x) \delta-\lambda\|_{1}+\|X \Lambda e-\mu e\|_{1}+\|c(x)\|_{1} \text { and } \Phi(x, \mu) \text { is the barrier function. }
$$

Using the filter methodology, a trial iterate $u_{k}\left(\alpha_{k}\right)=u_{k}+\alpha_{k} \Delta_{k}$ might be acceptable by the filter if it leads to sufficient progress in one of the two previously defined measures, when compared to the current iterate:

$$
\theta\left(u_{k}\left(\alpha_{k}\right)\right) \leq\left(1-\gamma_{\theta}\right) \theta\left(u_{k}\right) \quad \text { or } \quad \Phi\left(x_{k}\left(\alpha_{k}\right), \mu\right) \leq \Phi\left(x_{k}, \mu\right)-\gamma_{\Phi} \theta\left(u_{k}\right)
$$

where $\gamma_{\theta}, \gamma_{\Phi} \in(0,1)$ are fixed constants. However, to prevent convergence to a stationary but nonoptimal point, and whenever $\theta\left(u_{k}\right) \leq \theta_{\min }$ and for the trial step size $\alpha_{k}$, the following switching conditions

$$
m_{k}\left(\alpha_{k}\right)<0 \text { and }\left[-m_{k}\left(\alpha_{k}\right)\right]^{s_{o}}\left[\alpha_{k}\right]^{1-s_{o}}>\delta\left[\theta\left(u_{k}\right)\right]^{s_{e}}
$$


hold, with fixed constants $\delta>0, s_{e}>1, s_{o} \geq 1$, where $m_{k}(\alpha)=\alpha \nabla \Phi\left(x_{k}, \mu\right)^{T} \Delta x_{k}$, then the trial iterate must satisfy the Armijo condition

$$
\Phi\left(x_{k}\left(\alpha_{k}\right), \mu\right) \leq \Phi\left(x_{k}, \mu\right)+\eta m_{k}\left(\alpha_{k}\right),
$$

instead of (4), to be acceptable. Here, $\eta \in(0,0.5)$ is a constant.

At the beginning of the iterative process, the filter is initialized to $\bar{F}_{0} \subseteq\left\{(\theta, \Phi) \in \mathbb{R}^{2}: \theta \geq \theta^{\max }, \Phi \geq \Phi^{\max }\right\}$ for some positive constants $\theta^{\max }$ and $\Phi^{\max }$. Whenever the accepted step size satisfies (4), the filter is updated by

$$
\bar{F}_{k+1}=\bar{F}_{k} \cup\left\{(\theta, \Phi) \in \mathbb{R}^{2}: \theta \geq\left(1-\gamma_{\theta}\right) \theta\left(u_{k}\right) \text { and } \Phi \geq \Phi\left(x_{k}, \mu\right)-\gamma_{\Phi} \theta\left(u_{k}\right)\right\},
$$

and it remains unchanged if for the accepted step size, (5) and (6) hold. Finally, when the trial step size $\alpha_{k}$ becomes too small, the algorithm reverts to a feasibility restoration phase that aims to find a new iterate $u_{k+1}$ that is acceptable to the current filter, for which (4) holds, by solving

$$
\min _{x}\|c(x)\|_{1}+\frac{\varsigma}{2}\left\|D\left(x-x^{(k)}\right)\right\|^{2}, \text { subject to } x \geq 0
$$

where $D$ is a diagonal scaling matrix and $\varsigma>0$ is the weighting parameter. This aims to decrease the infeasibility and consequently the KKT error, and avoids large deviation from $x^{(k)}$. The reader is referred to $[11,12,13]$ for details.

\section{NUMERICAL EXPERIMENTS}

To analyze the performance of the proposed filter line search method, integrated into the IPOPT code, with modifications in some files of the directory "Ipopt/src/Algorithm", when compared with IPOPT itself, we used and run IPOPT version 3.5.5 with linear solver MA27. IPOPT was run with default options, namely the second order correction, see [13]. A set of 420 small to large constrained problems, with $n$ varying from 2 to 10000 (77 problems with $1000 \leq n \leq 10000$ ) from the CUTEr collection was used and the results were obtained in a computer Core 2 Duo T9550@2.66 GHz with 4 GB memory, running Linux Ubuntu 8.10.

To compare the performance of both filter line search strategies, we use the performance profiles as described in Dolan and Morés paper [4]. These profiles represent the cumulative distribution function for the performance ratio based on a chosen metric. Our profiles considered the following metrics: CPU time, number of iterations and number of objective function evaluations. Let $\mathscr{P}$ and $\mathscr{S}$ be the set of problems and the set of solvers in comparison, respectively, then we use $m_{p, s}$ to represent the performance metric required to solve problem $p \in \mathscr{P}$ by solver $s \in \mathscr{S}$. The comparison is based on the performance ratios defined by

$$
r_{p, s}=\left\{\begin{array}{l}
1+m_{p, s}-\min \left\{m_{p, s}: s \in \mathscr{S}\right\}, \text { if } \min \left\{m_{p, s}: s \in \mathscr{S}\right\}<\varepsilon, \\
\frac{m_{p, s}}{\min \left\{m_{p, s}: s \in \mathscr{S}\right\}}, \text { otherwise }
\end{array},\right.
$$

for $\varepsilon=0.00001$, and the overall assessment of the performance of a particular solver $s$ is given by

$$
\rho_{s}(\tau)=\frac{1}{n_{P}} \operatorname{size}\left\{p \in \mathscr{P}: r_{p, s} \leq \tau\right\}
$$

where $n_{P}$ is the number of problems in the set $\mathscr{P}$ and "size" is the number of problems in the set such that $r_{p, s}$ is less than or equal to $\tau$ for solver $s$. Thus, $\rho_{s}(\tau)$ gives the probability (for $s \in \mathscr{S}$ ) that $r_{p, s}$ is within a factor $\tau \in \mathbb{R}$ of the best possible ratio. The higher the $\rho$ the better the solver is.

We present in Figure 1 the profiles of the CPU times of both filter strategies. Our proposed filter line search is slightly more efficient than the original IPOPT. We do not show the profiles of the number of iterations and function evaluations because the numbers are equal. The percentage of robustness of both strategies is 96 .

\section{CONCLUSIONS}

In this paper, we presented a new filter line search method to integrate into a primal-dual barrier approach, for the efficient solving of nonlinear optimization problems. Each entry in the filter has two components measuring KKT error and optimality, being the latter based on the barrier function value. Computational experiments carried out with the herein proposed filter framework, when integrated into the IPOPT code, and compared with the original IPOPT, show that our strategy is rather competitive. We aim to contribute with this piece of code to IPOPT open source project. 


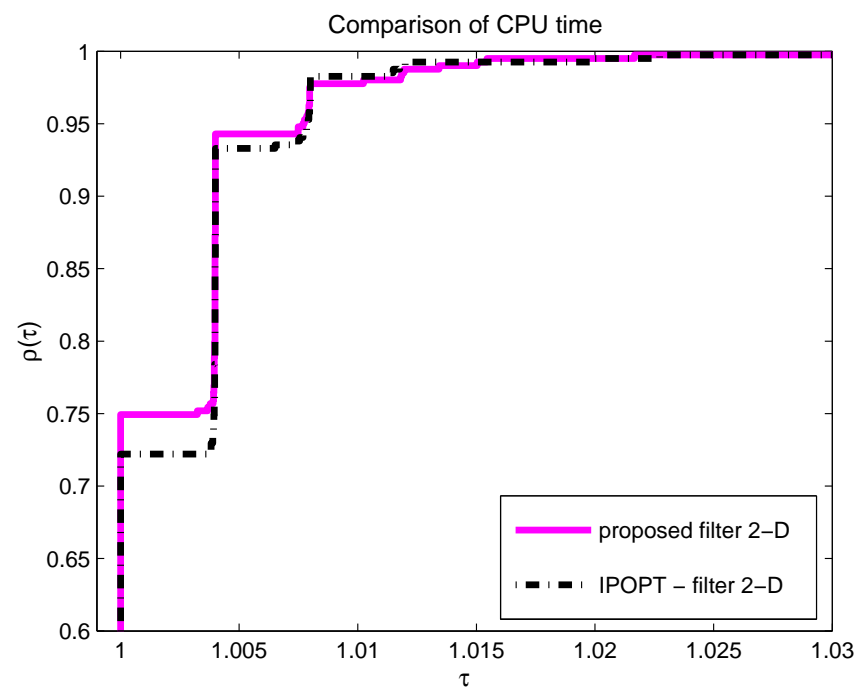

FIGURE 1. Performance profiles of CPU time

\section{ACKNOWLEDGMENTS}

This work has been partially supported by the FCT (Fundação para a Ciência e a Tecnologia), Portugal.

\section{REFERENCES}

1. M.F.P. Costa and E.M.G.P. Fernandes, Comparison of interior point filter line search strategies for constrained optimization by performance profiles, International Journal of Mathematics Models and Methods in Applied Sciences, 1, 2007, 111-116.

2. M.F.P. Costa, and E.M.G.P. Fernandes, Practical implementation of an interior point nonmonotone line search filter method, International Journal of Computer Mathematics, 85, 2008, 397-409.

3. M.F.P. Costa, and E.M.G.P. Fernandes, A three-D filter line search method within an interior point framework, Proceedings of 2008 ICMMSE, ISBN: 978-84-612-1982-7, 2008, 173-187.

4. E.D. Dolan and J.J. Moré, Benchmarking optimization software with performance porfiles, Mathematical Programming A, 91, 2002, 201-213.

5. R. Fletcher and S. Leyffer, Nonlinear programming without a penalty function, Mathematical Programming, 91, 2002, $239-269$.

6. R. Fletcher, S. Leyffer and P. Toint, A brief history of filter methods, Report ANL/MCS-P1372-0906, Argonne National Laboratory 2006.

7. N.I.M. Gould, D. Orban, A. Sartenaer and P.L. Toint, Superlinear convergence of primal-dual interior point algorithms for nonlinear programming, SIAM Journal on Optimization, 11, 2001, 974-1002.

8. D.F. Shanno and R.J. Vanderbei, Interior-point methods for nonconvex nonlinear programming: orderings and higher-order methods, Mathematical Programming B, 87, 2000, 303-316.

9. M. Ulbrich, S. Ulbrich and L.N. Vicente, A globally convergent primal-dual interior-point filter method for nonlinear programming, Mathematical Programming, 100, 2004, 379-410.

10. R.J. Vanderbei and D.F. Shanno, An interior-point algorithm for nonconvex nonlinear programming, Computational Optimization and Applications, 13, 1999, 231-252.

11. A. Wächter and L.T. Biegler, Line search filter methods for nonlinear programming: motivation and global convergence, SIAM Journal on Optimization, 16, 2005, 1-31.

12. A. Wächter and L.T. Biegler, Line search filter methods for nonlinear programming: local convergence, SIAM Journal on Optimization, 16, 2005, 32-48.

13. A. Wächter and L.T. Biegler, On the implementation of an interior-point filter line-search algorithm for large-scale nonlinear programming, Mathematical Programming, 106, 2007, 25-57. 